\title{
Sentimientos que experimentan los padres en el difícil camino de la hospitalización de sus hijos prematuros. Un aporte al cuidado humanizado
}

\author{
Feelings experienced by parents when their premature children are hospitalized. A contribution to the \\ humanized care
}

\author{
María Fernanda Acosta-Romo ${ }^{*}$ orcid.org/0000-0003-0772-8021 \\ Nancy Cabrera-Bravo² orcid.org/0000-0001-6985-1581 \\ Yudy Basante-Castro' ${ }^{1}$ orcid.org/0000-0002-9962-8572 \\ Daniel Jurado3,4 orcid.org/0000-0003-0628-0253
}

$\begin{array}{ll}1 & \text { Facultad de Posgrados y Relaciones Internacionales - Campo de la Salud, Universidad Mariana. Pasto, Colombia } \\ 2 & \text { Unidad de Cuidado Intensivo Neonatal Hospital Infantil los Ángeles. Pasto, Colombia } \\ 3 & \text { Grupo Investigación Salud Pública, Centro de Estudios en Salud Universidad de Nariño. Pasto, Colombia } \\ 4 & \text { Facultad Ciencias de la Salud, Programa de Medicina, Universidad de Nariño. Pasto, Colombia }\end{array}$ hospitalización de sus hijos prematuros. Un aporte al cuidado humanizado. Univ. Salud. 2017;19(1):17-25. D0I:

\section{Resumen}

Objetivo: Comprender el significado de las vivencias que experimentan los padres de niños prematuros hospitalizados en la Unidad de Cuidado Intensivo Neonatal de un hospital en la ciudad de Pasto, Nariño, a la luz de la teoría fenomenológica de Edmund Husserl. Materiales y métodos: Para comprender esas vivencias se realizó un estudio cualitativo, fenomenológico, con ocho padres. Los entrevistados se encontraban en un rango de edad entre 17 y 35 años. Resultados: El proceso investigativo permitió identificar cinco categorías, las cuales surgieron a partir del proceso de codificación o análisis nomotético de las convergencias de los discursos. Para el presente artículo se tomó dos de ellas: Sentimientos y vínculo afectivo como expresión de amor paterno y proceso de interacción con el personal de salud. Conclusión: Los padres de hijos hospitalizados no estaban preparados para el nacimiento de un niño prematuro, por lo que experimentaron sentimientos de tristeza, ansiedad, autocrítica y temor, alterándose el vínculo afectivo entre padres e hijos.

Palabras clave: Cuidado intensivo neonatal; emociones; padres; recién nacido prematuro. (Fuente: DeCS, Bireme).

\begin{abstract}
Objective: To understand the meaning of the experiences felt by parents of premature children who are hospitalized in the Neonatal Intensive Care Unit of a hospital in the city of Pasto, Nariño, taking into account the phenomenological theory of Edmund Husserl. Materials and methods: In order to understand these experiences, a qualitative phenomenological study was carried out with eight parents in a range of age between 17 and 35. Results: The investigative process identified five categories, which emerged from the process of codification or nomothetic analysis of the speeches convergences. Two of these categories were considered for this article: Feelings and affective bond as an expression of parental love and process of interaction with the health staff. Conclusion: The parents of children in hospital were not prepared for the birth of a premature baby, so they experienced feelings of sadness, anxiety, self-criticism and fear, altering the affective bond between parents and children.
\end{abstract}

Keywords: Neonatal intensive care; feelings; parents; preterm newborn baby. (Source: DeCS, Bireme). 


\section{Introducción}

Uno de cada diez niños nace prematuramente (nacido vivo antes de las 37 semanas de gestación), y cada año muere un millón de ellos por complicaciones; más de las tres cuartas partes de los recién nacidos prematuros pueden salvarse con medidas asistenciales factibles y costos eficaces ${ }^{1}$.

Actualmente, se observa que la supervivencia de los bebés prematuros es cada vez mayor, debido a los enormes progresos de la neonatología; sin embargo, estos avances no han sido suficientes debido a que el entorno de las Unidades de Cuidado Intensivo Neonatal (UCIN) generan en los padres de hijos prematuros emociones negativas (depresión, temor, culpabilidad, estrés, etc.) y con ellas surge una serie de preguntas y dudas. La hospitalización está siempre acompañada de una gran movilización emocional de la familia como un todo y más directamente de los padres. El nacimiento de un nuevo integrante de la familia normalmente simboliza alegría, celebración y expectativa, cuando algo interrumpe esta armonía, verdaderos dramas familiares son vivenciados ${ }^{2}$.

Durante la hospitalización del recién nacido, el profesional de salud brinda cuidado y es partícipe de la recuperación, e igualmente está en continuo contacto con los padres cuando ingresan a la unidad a visitar a su pequeño, de ahí la importancia que el equipo interdisciplinario de las UCIN trabajen con un método inductivo, enfocado en las personas y no únicamente en la enfermedad ${ }^{3}$.

En la UCIN la atención se centra en el estado de salud del niño, pero los padres también necesitan atención y ser escuchados, por consiguiente el personal de salud debe interesarse por conocer la forma como ellos están vivenciando la transición de tener un recién nacido hospitalizado en dicha unidad.

Con el objetivo de comprender el significado de las vivencias que experimentan los padres de niños prematuros hospitalizados en la UCIN desde una visión humanística, se decidió realizar la presente investigación de enfoque cualitativo con abordaje fenomenológico, el cual permite ir al mundo vivido o realidad vivida de los padres de hijos prematuros, a fin de traer a la luz la experiencia de esta situación y comprender las vivencias de ellos. En la fenomenología, lo más importante es el reconocimiento de la experiencia, porque a medida que se comparte una vivencia, una historia de vida, se pueden descubrir los significados ocultos y los fenómenos por las apariencias $\mathrm{y}$, por tanto, es posible llegar a las esencias originarias de los seres humanos ${ }^{4}$.

Como resultado de este proceso investigativo, se identificaron cinco categorías las cuales surgieron a partir del proceso de codificación o análisis nomotético de las convergencias de los discursos. Dichas categorías son: Sentimientos y vínculo afectivo como expresión de amor paterno, proceso de interacción con el personal de salud, la familia y la experiencia de la hospitalización, un mundo desconocido e inesperado: UCIN, presencia emocional y espiritual. Para el presente artículo se tomaron dos de ellas: sentimientos y vínculo afectivo como expresión de amor paterno y proceso de interacción con el personal de salud.

\section{Materiales y métodos}

\section{Tipo de Estudio}

Se realizó un estudio fenomenológico, apropiado para el caso, ya que se preocupa por los seres humanos y su ambiente en toda su complejidad. Para develar este fenómeno se basó en la corriente filosófica denominada fenomenología eidética, específicamente en la fenomenología de Edmund Husserl5.

\section{Muestra}

Se llevó a cabo entrevistas en profundidad a ocho personas que hicieron parte del estudio, la mayoría mujeres: siete madres y un padre. Los padres de hijos prematuros se encontraban en un rango de edad entre 17 y 35 años. 


\section{Criterios de inclusión}

Padres de niños prematuros hospitalizados en la UCIN que no presentan ninguna discapacidad mental, padres de recién nacidos con edad gestacional menor o igual a 34 semanas al nacer, y que además el tiempo de hospitalización fuese como mínimo un mes . El muestreo que se utilizó para escoger este grupo, fue no probabilístico o por conveniencia, de tipo intencional, en el cual las investigadoras tomaron a algunos padres de hijos prematuros hospitalizados en la UCIN que cumplían con las características mencionadas anteriormente6; se realizaron las entrevistas hasta lograr la saturación de la información en los discursos, se identificaron con códigos para preservar la confidencialidad. Así mismo, para la aplicabilidad y transferibilidad del estudio se sustentaron los resultados en diferentes ponencias.

\section{Recolección y análisis de la información}

Se realizó contacto inicial y sensibilización por medio del folleto "Carta de un prematuro a sus padres"7, aplicación de consentimiento informado y definición de primer encuentro. Posteriormente, se realizaron las entrevistas en profundidad, obteniendo información completa y profunda acerca del objeto de estudio, se efectuaron en forma individual. Además, se adaptó un guion predefinido teniendo como base preguntas orientadoras, las cuales fueron transcritas y analizadas a medida que se iban desarrollando las entrevistas, hasta que se saturó la información con los datos obtenidos.

El análisis de las mismas se hizo simultáneamente durante la etapa de recolección de la información, con el fin de monitorear permanentemente los datos y evitar información reiterativa, igualmente para identificar categorías analíticas potenciales a ser examinadas en la etapa de análisis. El análisis profundo de la información se efectuó una vez se consideró que los discursos daban respuesta a la pregunta de investigación planteada ${ }^{8}$.

Para el desarrollo de la investigación de trayectoria fenomenológica, se siguieron tres momentos: descripción, reducción y la comprensión.

Descripción: desde el momento que se escucha a los padres de hijos prematuros y hasta el final del análisis, de manera que se incorporó lo que ellos describieron en sus discursos, luego se reorganizó la totalidad del discurso en sus partes, lo cual permitió ver lo esencial en los diferentes momentos del análisis, que son: unidades de significado, reducción fenomenológica, agrupamiento de las unidades de significado e interpretación.

A medida que los investigadores se familiarizaron con las descripciones a través de repetidas lecturas, surgieron las unidades de significado, de tal forma que se sistematizó lo que es vivido por los padres de hijos prematuros hospitalizados en la UCIN. Posteriormente, se realizó la reducción fenomenológica, en este momento el objetivo fue determinar $\mathrm{y}$ seleccionar las partes de la descripción de los discursos que fueron consideradas esenciales y aquéllas que no lo fueron. Seguido a esto se dio paso a la Comprensión, en la cual se transformó las unidades de significado al lenguaje propio de los investigadores, con miras hacia la comprensión de la estructura individual de las vivencias de los padres de niños prematuros hospitalizados en la UCIN.

Cuando las descripciones de los discursos convergieron, se puedo establecer que el fenómeno en estudio se mostró y se llegó a la aprehensión de los significados esenciales. En el tercer momento de la trayectoria se construyó el análisis ideográfico, se tomó los discursos dados por los padres para la comprensión del fenómeno, realizando una reflexión que clarificó los puntos significativos del discurso. Y finalmente, se efectuó el análisis nomotético. Después de haber concluido los análisis individuales de todos los discursos se desarrolló un análisis de lo general, que es la búsqueda de generalidades del fenómeno interrogado ${ }^{9}$ (Figura 1). 


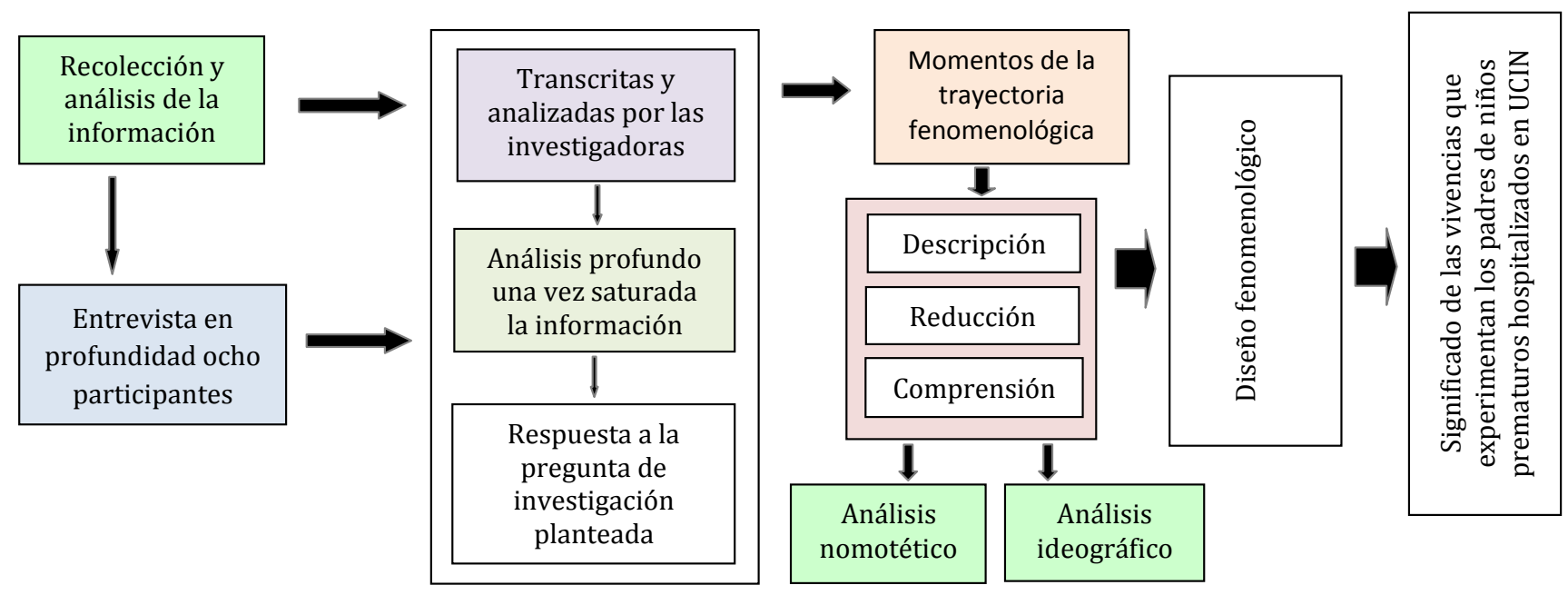

Figura 1. Proceso de Análisis de la información

\section{Consideraciones Éticas}

Para el desarrollo de este estudio se tuvo en cuenta la resolución 8430 de octubre 4 de 1993, en la cual se establecen las normas científicas, técnicas y administrativas para la investigación en salud en Colombia ${ }^{10}$. Así mismo, se respetaron los principios éticos contemplados en la declaración de Helsinki11. El estudio contó con la aprobación del Comité Ética y Bioética de la Universidad Mariana y el Hospital Infantil los Ángeles.

\section{Resultados}

A continuación se presentan dos de las categorías tituladas: Sentimientos y vínculo afectivo como expresión de amor paterno y proceso de interacción con el personal de salud.

\section{Sentimientos y vínculo afectivo como expresión de amor paterno}

El tener un hijo prematuro es un evento no esperado por los padres, ya que ellos no contemplan dentro del nacimiento esta situación, por tanto, no se encuentran preparados para asumirlo. Por lo general, los niños prematuros requieren ser hospitalizados en la UCIN debido a su condición clínica; esta experiencia es más difícil de asumir, ya que el ambiente de la sala de cuidado intensivo neonatal genera sentimientos en los padres, como lo describieron en los siguientes discursos:

(...) es muy duro para mí como madre separarme de mi bebé porque es muy pequeño y muy frágil (...). $\left(D_{1}\right)$.

(...) Sentí angustia; no me la dejaron ni ver, ni tocar. $\left(D_{2}\right)$.

Para mí es bien difícil porque uno al mirarla ahí, se llena de nostalgia y uno quiere como cogerla, abrazarla y eso no se puede. (...). ( $\left.D_{4}\right)$.

Este estudio muestra que la separación de un hijo es una experiencia difícil para los padres, de ahí la importancia de que el personal de salud de la UCIN implemente estrategias que permitan la vinculación y participación de los padres en el cuidado del recién nacido prematuro durante la hospitalización, más aún cuando son estancias prolongadas.

Por otra parte, la interrupción en el vínculo afectivo madre-hijo, genera un impacto negativo en las madres, quienes manifiestan sentimientos como: miedo, tristeza y angustia; éstos se ven agravados por las condiciones clínicas del recién nacido. Esta experiencia se refleja en:

Siento un miedo muy grande tenerlo aquí (...). $\left(D_{1}\right)$. 
(...) Angustiado (...) porque igual está pequeño todavía y no sabemos cómo vaya evolucionando de aquí en adelante. (...). ( $\left(\mathrm{D}_{3}\right)$.

(...) tristeza porque yo esperaba tener un parto normal y mirarlo que esté sanito (...), tristeza, preocupación. $\left(\mathrm{D}_{5}\right)$.

Yo sentía miedo de que de pronto la niña fuera a decaer, que se fuera a morir (...) a veces siento alegría otras veces tristeza (...). $\left(\mathrm{D}_{6}\right)$.

La autocrítica -culpabilidad- es otro sentimiento que aflora en los padres, especialmente en la madre, por la fragilidad de su hijo y la situación clínica del prematuro; ella piensa que los cuidados durante su embarazo no fueron suficientes y por consiguiente no se logró que éste llegara a feliz término:

(...) Durísimo porque uno se empieza a echar la culpa de todos los meses anteriores (...) no podía comer y por eso creo que la niña es flaquita. (...). Decía por mi culpa la niña está así. (Llanto). ( $\left.\mathrm{D}_{4}\right)$.

(...) Culpa porque si me hubiera cuidado más... (Llanto) en mi embarazo y todo, de pronto $\mathrm{mi}$ bebé no hubiera nacido tan pequeñito y hubiera logrado más semanas de gestación. $\left(\mathrm{D}_{8}\right)$.

(...) culpa porque me dijeron que no me podía embarazar antes de 2 años; debía recibir tratamiento; si, yo creo por irresponsabilidad, porque uno hace las cosas sin pensar y cuando uno se da cuenta ya es tarde. $\left(\mathrm{D}_{2}\right)$.

\section{Proceso de interacción con el personal de salud}

La UCIN es un lugar que ofrece servicios de alta complejidad debido al estado crítico de los recién nacidos; por este motivo los padres se encuentran sometidos a diferentes cambios y necesidades, que son importantes de identificar para intervenir y dar una solución adecuada, de manera que se pueda facilitar el acercamiento de los padres con su hijo recién nacido hospitalizado. La comunicación hace parte del cuidado; además de brindar una atención clínica, se debe tratar de identificar las necesidades en los padres, ya que para ellos la comunicación se convierte en algo prioritario. Sin embargo, a menudo hay falta de información en esta unidad como lo manifiestan algunos de ellos:

\section{(...) si yo no preguntaba, no me decían nada. ( $\left.D_{5}\right)$.}

(...) a veces utilizan términos que uno no entiende. $\left(D_{6}\right)$.

(...) uno se siente preocupada, no sabe (...) lo que le dicen los médicos (...) porque no entiendo la explicación, o sea, que le digan a uno la verdad,(...) cuando le dicen las cosas claras, la realidad de lo que pasa, entonces uno se siente con más seguridad (...). ( $\left.\mathrm{D}_{7}\right)$.

Me han informado acerca de la salud de mi hijo; primero la enfermera jefe, y luego el pediatra, me van explicando cómo va evolucionando nuestro bebé $(\ldots) .\left(\mathrm{D}_{1}\right)$.

Algunos discursos de los padres, demuestran que el equipo de salud brinda información acerca del estado clínico de sus hijos, esto facilita un acercamiento con ellos y de esta manera, los padres se convierten en parte fundamental y esencial en el cuidado de sus prematuros; lo cual ayuda en gran parte a reducir el estrés que genera la hospitalización, como lo evidencian en las siguientes expresiones:

La comunicación con el personal de salud por lo general es buena, (...) yo les pregunto y me dan información. (...). ( $\left.\mathrm{D}_{5}\right)$.

Me han dado información, me ha parecido un servicio excelente; están pendientes de mi bebé todas las personas que están acá, me dan la información que yo pido y si no entiendo me la vuelven a explicar, (...) $\left(\mathrm{D}_{8}\right)$.

\section{Discusión}

Ante la inquietud de develar el fenómeno o las experiencias que experimentan los padres de hijos prematuros hospitalizados en la UCIN, se realizó esta investigación, la cual pone de manifiesto que los padres son las personas que directamente se ven implicados con el proceso de hospitalización de un hijo prematuro; esta situación genera en ellos diversos sentimientos, como: culpa -autocrítica-, preocupación, temor por el desenlace y la apariencia física del recién nacido, también manifiestan sentir ansiedad, por la situación de riesgo en la que se encuentra su hijo. Este es un tema que converge en otros estudios, la ansiedad es la emoción negativa principal ${ }^{12}$. 
Es preciso decir que, los padres necesitan estar junto a su bebé para sentirse seguros; si este vínculo se interrumpe, afecta negativamente el estado emocional madre-hijo. Los pediatras Klaus y Kennell citados por Guerra ${ }^{13}$, proponen la hipótesis que sugiere: "Existe, inmediatamente después del parto, un período durante el cual el apego de la madre hacia su hijo se desarrolla. Durante este periodo tan particular, cuya duración se estima entre pocos minutos y pocas horas, la probabilidad de que ciertos acontecimientos den origen a los lazos afectivos madre-hijo será más fuerte en este período que en cualquier otro momento de la vida del niño. Más específicamente, entre la madre y el hijo se desarrollan interacciones complejas y crean una unión permanente entre ellos". Este estudio muestra que la separación de un hijo es una experiencia difícil para los padres, de ahí la importancia de que el personal de salud de la UCIN, implemente estrategias que permitan la vinculación y participación de los padres en el cuidado del recién nacido prematuro durante la hospitalización, más aún cuando son estancias prolongadas.

Algunos padres manifiestan sentir dolor por la separación de su hijo, esta situación puede deberse a factores financieros, horarios restringidos de visita, entre otros ${ }^{14}$. En relación con los resultados del presente estudio, se puede mencionar que la separación también afecta a los padres, pero las causas no son convergentes, debido a que en la institución prestadora de salud (IPS) en la cual se realizó el estudio, la unidad de cuidado intensivo no tiene restricción en su ingreso; asimismo, se menciona como una situación muy dura el hecho de no haber tenido la oportunidad del apego inmediato con su hijo, lo anterior puede ser debido a que la IPS ejecutora del estudio no cuenta con sala de atención de partos, por tanto, los neonatos hospitalizados fueron remitidos de otros hospitales, afectando negativamente el vínculo afectivo madre e hijo.

Una de las estrategias que se ha implementado para restablecer y fortalecer la relación madrehijo es el Programa Madre Canguro, por medio del cual se ayuda a las madres o padres de hijos prematuros a fortalecer la seguridad y confianza en sí mismos e influye favorablemente disminuyendo su temor y ansiedad. Ésta es una estrategia apropiada para eliminar la separación entre padres e hijos y cambiar la vivencia de otros padres que pueden llegar a pasar por esta difícil situación. Además, ha demostrado que es posible reducir la crisis producida por el nacimiento anticipado y promueve el apego y el desarrollo del rol de cuidadores al que tienen derecho a ejercer los padres; de ahí, la importancia de que las UCIN cuenten con este programa $^{15}$.

Otro sentimiento que afloró en los padres fue la autocrítica o culpabilidad, al respecto los psicólogos mencionan que esta emoción se acerca más, a un sentimiento de vergüenza, sentimiento que se refiere a las consecuencias desagradables del pecado, más que al pecado en sí mismo ${ }^{16}$.

Aunque no surgieron en los padres expresiones de vergüenza, el sentimiento de culpa es una de las emociones que las personas experimentan en mayor o menor grado por algo que se hizo o se dejó de hacer; ante esto, es importante que el personal de enfermería esté atento a la expresión de estos sentimientos, como lo considera la doctora Jean Watson citada por Rivera, et al., "la enfermería es un arte, cuando la enfermera experimenta y comprende los sentimientos del otro, es capaz de detectar y sentir estos sentimientos y a su vez, es capaz de expresarlos, de forma semejante que la otra persona los experimenta" 17 . Cuando la enfermera identifica los sentimientos que están vivenciando los padres de hijos prematuros, ella se vuelve más sensible, siendo capaz de permitir la expresión de ellos y convertirse en una persona que escucha y canaliza estas expresiones hacia el logro de un ambiente de tranquilidad y confianza mutua; la humanización de los cuidados hace referencia a personas en interacción, que saben clarificar sus necesidades, reconocen sus sentimientos y se enfocan positivamente ${ }^{18}$.

Ahora bien, la UCIN es un lugar que ofrece servicios de alta complejidad, debido al estado crítico de los recién nacidos; por este motivo, los 
padres se encuentran sometidos a diferentes cambios y necesidades, las cuales son importantes identificar y poder intervenir para dar una solución adecuada y así facilitar el acercamiento de los padres con su hijo recién nacido hospitalizado para entender la situación clínica del recién nacido prematuro, la madre necesita información continua. Una de las necesidades que plantea Virginia Henderson es la comunicación con los otros; expresar sus pensamientos, sentimientos y emociones, interactuando con el resto de las personas y su entorno. Las emociones están íntimamente ligadas a las alteraciones de salud tanto física como psicológica. La Enfermería promueve el bienestar del paciente, fomentando las relaciones y la aceptación de la propia persona. En este sentido, este personal debe valorar el equilibrio entre la soledad - interacción social, estado de los órganos de los sentidos, capacidad de expresión, relaciones con la familia, los amigos y la pareja ${ }^{19}$.

Por la literatura revisada no es fácil comprender las vivencias de los padres de hijos prematuros, con el presente estudio se confirma la necesidad de brindar un cuidado integral, por tanto resulta importante sensibilizar a médicos, enfermeras, fisioterapeutas y en general a todo el equipo a prestar dentro de esta área un cuidado humanizado; el cual se ha convertido en una necesidad social. Para Stein et al., citado por Beltrán, resulta trascendente impulsar procesos de humanización centrado en el paciente, revisar los valores, sentimientos y actitudes que guían el modo de ser de los profesionales con el fin de proporcionar un servicio diferenciado $y$ humanizado, es decir, crear una cultura organizacional centrada en el ser humano enfermo y no en la enfermedad" 20 . Por tanto, se requiere que los profesionales que laboran en las UCIN, posean cualidades especiales como sensibilidad para percibir y comprender los sentimientos que experimentan los padres de hijos prematuros que requieren de su atención y de esta manera humanizar su accionar ${ }^{21}$, entender los efectos de un evento tan devastador como éste, es una necesidad para quienes laboran en el área de la salud, especialmente en la UCIN, en la que se realizan los cuidados como objeto de ellos no solo al paciente (neonato), sinó también a su grupo familiar ${ }^{22}$.

Los resultados del presente estudio evidenciaron que algunos de los padres han puesto de relieve el valor de optimizar la información y/o comunicación, apoyo y acompañamiento por parte de personas capacitadas con el fin de disminuir el estrés y la ansiedad que genera este tipo de situación ${ }^{23}$. La comunicación es un componente frecuentemente desatendido en la formación de los profesionales de la salud, pero es una habilidad que puede y debe ser enseñada y aprendida. Es esencial que los integrantes del equipo de salud sean capaces de comunicarse en forma abierta y compasiva con los padres y la familia del neonato ${ }^{24}$.

La enfermera Merle Mishel citada por Martínez, et al. ${ }^{13}$, afirma que si los padres poseen la información necesaria, pueden crear estrategias para ayudar al afrontamiento positivo de sus hijos durante el proceso de hospitalización. Para ello, conceptualiza el nivel de incertidumbre frente a la enfermedad como la incapacidad de la persona de determinar el significado de los hechos relacionados con la enfermedad, siendo incapaz de dar valores definidos a los objetos o hechos, y por lo tanto, no puede predecir los resultados, todos los fenómenos motivados por la falta de información y conocimiento.

Al respecto, en un estudio realizado por Espinosa et al., donde el objetivo fue construir participativamente el concepto de cuidado humanizado entre los investigadores y los sujetos de investigación se obtuvo como resultado final: "Es la expresión del acto de cuidado en la que se concibe integralmente al individuo, la familia y la colectividad; en la que se reconoce la dignidad y condición humana del otro; en la que se respetan los principios éticos; en la que se reflexiona acerca de sus necesidades de cuidado en salud y de sus respuestas a las mismas para asistirlo en aquellas demandas en las que se encuentre deficitario, promoviendo dentro de las posibilidades la recuperación pronta de su autocuidado a través de una comunicación asertiva y efectiva" 25 . 
El anterior concepto pone de manifiesto la importancia de la comunicación entre personal de salud y los sujetos de cuidado, la falta de información puede tener una influencia negativa en la calidad de la prestación de los servicios, ya que la comunicación debe hacer parte del cuidado humanizado. Además, de brindar una atención clínica, se debe tratar de identificar las necesidades de los padres y la familia del neonato, sin embargo a menudo hay falta de información, así lo manifiestan los padres en algunos de sus discursos.

Los padres necesitan ser bien recibidos y atendidos de forma individualizada, el personal de salud debe buscar el momento oportuno para brindar una información y/o comunicación asertiva, continua, acerca del estado de salud del neonato, su evolución, tratamiento y pronóstico, mostrando una actitud de comprensión y apoyo ${ }^{26}$, de esta manera, también se estaría contribuyendo a mitigar una de las necesidades más relevantes que tienen los padres de hijos prematuros hospitalizados en la UCIN.

Poco a poco, la mirada del personal de salud se debe dirigir a los padres de los niños hospitalizados en la UCIN para reconocerlos y darles el espacio que tienen por derecho propio al lado de sus hijos, y ofrecer acciones de cuidado que les proporcionen elementos adaptativos que indudablemente van más allá de dar información sobre la enfermedad del bebé y los procedimientos realizados ${ }^{27}$; situación que facilita un acercamiento, convirtiendo a los padres en parte fundamental y esencial en el cuidado de sus hijos prematuros y a su vez que alivia en parte el estrés que genera la hospitalización.

Por lo mencionado, es importante resaltar que a la luz de la teoría del cuidado humano de Jean Watson citada por Rivera, et al., "La empatía, que hace referencia a todas aquellas actitudes $\mathrm{y}$ acciones positivas que surgen en la interacción de cuidado como: escuchar, comprender lo que el otro siente, $y$ ponerse en su lugar transpersonalizar, genera la facilidad $y$ disponibilidad para acercarse al otro, tener tiempo para escucharlo, comunicarse amablemente y establecer una relación empática; es la capacidad de experimentar, y por lo tanto, de comprender las percepciones y sentimientos de otra persona, así como la capacidad para comunicar esta comprensión"12. Algunos de los padres entrevistados manifestaron recibir una buena información, contribuyendo así al acercamiento y confianza de los padres con el personal de salud, logrando una mejor atención para el éxito en la recuperación del recién nacido.

\section{Conclusiones}

Los padres no se encontraban preparados para el nacimiento de un hijo prematuro, por lo cual experimentaron diferentes sentimientos: tristeza, angustia, autocrítica y temor ante la hospitalización de su hijo, retrasando el establecimiento de los lazos paternales.

La comunicación hace parte del cuidado de Enfermería, además de brindar una atención clínica, se debe tratar de identificar las necesidades de los padres, ya que para ellos la comunicación oportuna y asertiva se convierte en algo prioritario dentro de la Unidad de Cuidado Intensivo Neonatal.

Los hallazgos de esta investigación sirvieron como evidencia para ratificar que el personal de salud que labora en las UCIN tiene la responsabilidad de brindar cuidado desde el enfoque biologista, pero no debe ser su centro de atención, también deben enfocarse en brindar un cuidado humanizado a partir de las percepciones, con visión de las personas de manera holística.

Conflicto de intereses: Ninguno declarado por los autores.

\section{Referencias}

1. Organización Mundial de la Salud. Estadísticas Sanitarias Mundiales 2013 [En línea]. Ginebra: OMS; 2013. [citado 13 jul 2013]. Disponible en: http//www.unicef.org/spanisch/infobycountry/colom biasatistics. 
2. Argote L, Montes M. La donación hace la diferencia en el cuidado de padres y niños prematuros: Relación padres e hijos. Grupo Família, Fam Saúde Desenv. 2002; 4(1):7-15.

3. Vélez C, Vanegas, J. El Cuidado En Enfermería Perspectiva Fenomenológica. Hacia La Promoción de la Salud. 2011;16(2):175-189.

4. Do Prado M, De Souza M, Monticelli M, Cometto $M$, Gómez P. Investigación cualitativa en enfermería. Metodología y Didáctica. Washington: OPS, OMS; 2013.

5. Lambert C. Edmund Husserl: La Idea de la Fenomenología. Teología y Vida [En línea]. 2006 [citado 22 Ene 2016]; 47(4):517-529. Disponible en: https://dx.doi.org/10.4067/S004934492006000300008 .

6. Vasilaches I. Estrategia de investigación cualitativa. Barcelona: Gedisa SA; 2006.

7. Somos múltiples. Querida madre de un bebe prematuro. [En línea]. España: Somos múltiples. 2016 [Citado 20 feb 2013]. Disponible en: http://www.somosmultiples.es/blog/2014/11/17/ma dre-bebe-prematuro/

8. Do Prado ML, De Souza ML, Carraro Telma E. Investigación Cualitativa en Enfermería: contexto y bases conceptuales. Serie PALTEX Salud y Sociedad. Washington: OPS, OMS; 2008. P. 103-108.

9. Pineda E, De Alvarado E. Metodología de la investigación. Washington: Organización Panamericana de la Salud; 2008. p. 106-108.

10. Ministerio de la Protección Social. Resolución 008430 de 1993. Santafé de Bogotá: Presidencia de la República; 1993.

11. Declaración de Helsinki de la Asociación Médica Mundial. Principios éticos para las investigaciones médicas en seres humanos. [En línea]. Brasil: AMM; 2013 [citado 22 Ene 2016] Disponible en: http://www.wma.net/en/30publications/10policies/b 3/index.html

12. Guerra M. Expresiones y Comportamientos de apego Madre Recién Nacido hospitalizado en la Unidad de Cuidado Intensivo. [Tesis de Maestría]. Bogotá: Facultad de enfermería, Universidad Nacional de Colombia; 2013.

13. Martínez $\mathrm{P}$, Morales $\mathrm{M}$, Piza E. Incertidumbre de los padres frente al postoperatorio inmediato de sus hijos en la Clínica Universitaria Teletón. [Tesis de Maestría]. Bogotá: Facultad de Enfermería, Universidad de La Sabana; 2010.

14. Lazaroso M, Ospina J, Riaño A. Programa madre canguro: una alternativa sencilla y costo eficaz para la protección de los recién nacidos prematuros o con bajo peso. Salud Pública. 2012;14(2):32-45.

15. Iriarte A, Carrion M. Experiencias de los padres de grandes prematuros en la Unidad de Cuidado Intensivo Neonatal: revisión sistemática de la evidencia cualitativa. Metas de Enfermería. 2013;16(2):20-25.

16. Elders L. El sentimiento de culpabilidad según psicología. La literatura y la filosofía moderna. [En línea] Madrid: Servicio de Publicaciones de la Universidad de Navarra; 1983 [Citado 20 sep 2014].
Disponible

en:

http://dadun.unav.edu/handle/10171/5794

17. Rivera N, Triana A. Cuidado Humanizado de Enfermería. Visibilizando la Teoría y la Investigación en la Práctica en la Clínica del Country. Actual Enferm. 2007; 10(4): 15-21

18. Romero E, Contreras I, Moncada A. relación entre cuidado humanizado por enfermería con la hospitalización de pacientes. Hacia La Promoción De La Salud [Internet]. 2016 Jun [citado 20 Dic 2016]; 21(1):26-36. Disponible en: http://www.scielo.org.co/pdf/hpsal/v21n1/v21n1a03. pdf

19. Henderson V. Fundamentos históricos y teóricos de enfermería. Modelos y cuidados [En línea]. [citado 3 octubre 2014]. Disponible en: https://www5.uva.es/guia_docente/uploads/2012/.../

20. Beltrán 0 . The meaning of humanized nursing care for those participating in it: Importance of efforts of nurses and healthcare institutions. Invest Educ Enferm. [Internet] 2016 [citado 20 Dic 2016]; 34(1):18. Disponible https://dx.doi.org/10.17533/udea.iee.v34n1a03

21. Muñoz M. La relación entre el equipo de salud y la familia del niño muriente. Acta Bioethica. 2011;17(2): 247-256.

22. Vargas R. Cuidado humanizado: Al paciente críticamente enfermo: Enfermería pieza clave en la atención. Ciencia y Cuidado. 2007; 4(4): 21-27.

23. Marques L, De Souza C, Castelo R, Pereira V. Vivências paternas durante a hospitalização do recém-nascido prematuro na Unidade de Terapia Intensiva Neonatal. Rev. bras. Enferm. 2012;65(5):788-794.

24. Uriarten A, Vila J, Pomares Y, Prieto A, Capote J. La familia y la limitación del esfuerzo terapéutico en los cuidados intensivos pediátricos. Aspectos éticos. Medisur. 2014; 12(1):16-23.

25. Espinosa A, Enríquez C, Leiva F, López M, Castañeda L. Collective building of a concept in nursing humanized care. Cienc. enferm. [Internet]. 2015 Ago [Citado 20 Dic 2016]; 21(2):39-49. Disponible en: http://www.scielo.cl/scielo.php?script=sci_arttext\&pid $=\mathrm{S} 0717$ 95532015000200005\&lng=en.http://dx.doi.org/10.40 67/S0717- 553201500020000.

26. De Cássia R, De Oliveira I, Cardoso C. La voz de la mujer-madre de prematuro en la unidad neonatal: un enfoque fenomenológico. Online braz j nur. 2014;13 (2):198-206.

27. Ocampo M. El hijo ajeno: vivencia de madres de niños prematuros hospitalizados. AQUICHAN. 2013;13(1):6980. 